\title{
Frontotemporal Lobe Degeneration as Origin of Scans Without Evidence of Dopaminergic Deficit
}

\author{
Manuel Menéndez-González ${ }^{1,2,3}$, Tania Álvarez-Avellón ${ }^{4}$, José M. Salas-Pacheco ${ }^{5}$, \\ Benito de Celis-Alonso ${ }^{6}$, Kathryn A. Wyman-Chick ${ }^{7}$ and Oscar Arias-Carrión ${ }^{8 *}$
}

${ }^{1}$ Servicio de Neurología, Hospital Universitario Central de Asturias, Oviedo, Spain, ${ }^{2}$ Instituto de Investigación Sanitaria del Principado de Asturias, Oviedo, Spain, ${ }^{3}$ Departamento de Morfología y Biología Funcional, Universidad de Oviedo, Oviedo, Spain, ${ }^{4}$ Departamento de Psicología, Universidad de Oviedo, Oviedo, Spain, ${ }^{5}$ Instituto de Investigación Científica, Universidad Juárez del Estado de Durango, Durango, México, ${ }^{6}$ Facultad de Ciencias Físico Matemáticas, Benemérita Universidad Autónoma de Puebla, Puebla, México, ${ }^{7}$ Regions Hospital/Health Partners Neuroscience Center, Saint Paul, MN, United States, ${ }^{8}$ Unidad de Trastornos del Movimiento y Sueño/Centro de Innovación Médica Aplicada, Hospital General Dr. Manuel Gea González, Ciudad de México, México

OPEN ACCESS

Edited by: Miguel Coelho,

Universidade de Lisboa, Portugal

Reviewed by:

Gennaro Pagano, King's College London, United Kingdom

Pedro J. Garcia-Ruiz,

Hospital Universitario Fundación Jiménez Díaz, Spain

*Correspondence: Oscar Arias-Carrión arias@ciencias.unam.mx

Specialty section:

This article was submitted to Movement Disorders, a section of the journal Frontiers in Neurology

Received: 11 January 2018 Accepted: 26 April 2018 Published: 24 May 2018

Citation:

Menéndez-González M, ÁlvarezAvellón T, Salas-Pacheco JM, de Celis-Alonso B, Wyman-Chick KA and Arias-Carrión O (2018)

Frontotemporal Lobe Degeneration as Origin of Scans Without Evidence

of Dopaminergic Deficit.

Front. Neurol. 9:335.

doi: 10.3389/fneur.2018.00335
The term scans without evidence of dopaminergic deficit (SWEDD) can be associated with any patient diagnosed at first with Parkinson's disease but with a negative dopamine transporter-single photon emission computed tomography (DaTSPECT), which does not confirm the presynaptic dopaminergic deficiency. Therefore, an alternative diagnosis should be sought to support parkinsonism as a clinical diagnosis. Parkinsonism is a well-known manifestation of frontotemporal lobar degeneration (FTLD), particularly frequent in those with positive DaTSPECT. Here, we reinforce previous observations that parkinsonism can be present in FTLD patients with negative DaTSPECT and therefore, FTLD may account for a percentage of patients with SWEDD. We gather the clinical observations supporting this hypothesis and describe a case report illustrating this idea. Studies suggest the result of DaTSPECT in FTLD may depend on the neuropathology and clinical subtype. However, most studies do not provide a clinical description of the clinical subtype or pathological features making the association between subtypes of FTLD and DaTSPECT results impossible at the moment. Further studies correlating clinical, neuropsychological, neuroimaging, genetic, and pathology findings are needed to better understand parkinsonism in FTLD.

\footnotetext{
Keywords: scans without evidence of dopaminergic deficit, parkinsonism, dopamine transporter-single, photon emission computed tomography, presynaptic dopaminergic deficiency, frontotemporal lobar degeneration
}

\section{INTRODUCTION}

Parkinson's disease (PD) is a common neurodegenerative disorder. It is characterized by progressive degeneration of dopaminergic neurons in the pars compacta of the substantia nigra and the loss of nerve terminals in the basal ganglia structures $(1,2)$. The dopaminergic system is one of the most studied neurochemical systems because damage to nigrostriatal neurons is the most critical component in the pathophysiology of PD $(1,3)$. Clinically it is manifested by the so-called "parkinsonian syndrome," consisting of extrapyramidal signs, including bradykinesia and at least one of the following: 4-6 Hz rest tremor, muscular rigidity and postural instability. The term "parkinsonism" 
encompass some nosologic entities, besides $\mathrm{PD}$, which are grouped by their shared clinical features but are separated by their different pathologies.

The role of nigrostriatal dopamine deficits in PD has been firmly established (4-6). In recent years, dopamine transporter-single photon emission computed tomography, named DaTSPECT, has been used to detect degeneration of presynaptic dopamine receptors in the nigrostriatal structures $(6,7)$. The active ingredient of [123I]FP-CIT SPECT is a cocaine analog, 123I-nortropane, labeled: $N$-u-fluoropropyl 2b-carbomethoxy-3b-(4-iodophenyl), also referred to as: ([123I]ioflupane). It binds to striatal presynaptic dopamine transporter (DaT) in animals and humans and helps visualize indirectly these neurons with SPECT. DaT is located on the plasma membrane, mainly of nerve terminals of dopaminergic neurons in the brain, particularly in the globus pallidus, cingulate cortex, amygdala, olfactory tubercle, and midbrain but specially in the striatum and nucleus accumbens. DaT reuptakes dopamine from the synaptic cleft into presynaptic neurons playing an important role in the buffering of this neurotransmitter (8).

Baseline DaT imaging has a very high negative predictive value for degenerative conditions affecting the nigrostriatal pathway. Only a negligible proportion of normal dopaminergic SPECT can be found in atypical parkinsonisms (9). The acronym SWEDDs (scans without evidence of dopaminergic deficits) (10), arose from the clinical trial literature of $\mathrm{PD}$, in which patients were imaged with $18 \mathrm{~F}$-dopa PET or DaTSPECT to monitor disease progression, revealing that a substantial proportion of clinically diagnosed cases of PD had regular nuclear medicine scans (4-15\%) and were, therefore, designated as SWEDDs $(10,11)$. Thus, the term SWEDDs can be associated with any patient diagnosed at first with PD but in which subsequent functional imaging did not confirm the presynaptic dopaminergic deficiency, although the use of the term SWEDD is itself controversial $(9,12)$.

The true etiology of the symptoms experienced by patients with SWEDD remains controversial, and it has been suggested that these individuals may represent a non-PD related movement disorder $(10,13,14)$. When patients with SWEDD were initially discovered, it was hypothesized that they might be within a prodromal phase of PD (15). However, subsequent research has demonstrated significant differences between patients with dopamine-deficient scans and patients with SWEDD. Patients with SWEDD lack response to levodopa (16) and do not demonstrate deficits in olfaction as frequently as patients with dopamine-deficient PD (17). Patients with SWEDD also have more significant cardiovascular and thermoregulatory dysfunction, orthostatic hypotension, sleep disturbances, and higher frequencies of daytime sleepiness than dopamine-deficient PD patients $(18,19)$. Although, patients with SWEDD can present motor features similar to those which are dopamine-deficient (PD), previous longitudinal research suggests that patients with SWEDD do not demonstrate the progression of motor symptoms (20) and continue to have normal DaTSPECTs for up to 4 years after they are initially identified $(19,21,22)$. In a 5-year follow-up study of 16 patients with SWEDD, only 2 patients demonstrated reduced dopamine uptake on DaTSPECTs, while 14 remained classified as SWEDD (11). These studies seem to indicate that individuals with SWEDD have a distinctly different disorder than dopamine-deficient PDs $(14,15,18,22)$. Moreover, patients with SWEDD and long-standing parkinsonism exhibit non-motor features that differ from those of patients with PD. SWEDD patients had worse mood and cardiovascular function and better olfactory function than PD patients, but remain similar to patients with SWEDD and alternative final diagnoses (19). In general, the term SWEDD is a useful approach for those patients with slow-progressing parkinsonism, with mild evolution compared with PD. Some frontotemporal lobar degeneration (FTLD) have parkinsonism and a mild clinical course, probably this subgroup could represent a minority percentage of SWEDD (23-26).

In a prospective study of our research group, 30 patients with hard-to-diagnose tremor and normal DaTSPECT were followed for 2 years (27). After the follow-up scan diagnosis was reached for 18 cases. The other 12 patients underwent a second DaTSPECT and were then followed for 12 additional months. After this, the clinical diagnosis was performed again. The final diagnoses included a list of different entities, including neurodegenerative and non-neurodegenerative disorders. However, for six patients diagnosis remained uncertain. Interestingly, these six patients developed cognitive impairment with outstanding frontal features. It was first speculated that although some patients with SWEDDs had, in fact, dystonic tremor or other well-known neurological conditions, it was conceivable that some patients might have suffered from a disorder that had not yet been described. It was speculated that some of these patients might have suffered a neurodegenerative process originated in the frontal cortex spreading to subcortical structures (versus the subcortical $\rightarrow$ cortical process present in PD). In this article, we present a case report illustrating this possibility and discuss the clinical, neuropsychological, genetics, and neuroimaging findings supporting the observation that FTLD is behind some cases of SWEDD.

\section{CASE REPORT}

Written informed consent was obtained from the patient for the publication of this case report. She is a 70-year-old woman who worked as a childcare worker and is now retired. She used to drink $30 \mathrm{~g}$ alcohol/day until 10 years ago and she still smokes 5 cigarettes/day. She was diagnosed with depressive syndrome 10 years ago. Currently, she is on Trazodone $100 \mathrm{mg} /$ day and Clonazepam $0.5 \mathrm{mg} /$ day. There are cases of PD (brothers) and depression (mother and maternal grandmother) among her family records.

She first visited neurology clinics 10 years ago with memory loss complaints. On that occasion, cognitive screening tests, blood tests, and CT scan were normal. Complaints were supposed to be in relation with a mood disorder, and no treatment was prescribed. She came again 5 years ago due to impairment of memory. She also complained about unbalanced gait, intentional tremor, and difficulty doing fine tasks. Caregivers referred social, behavioral changes, and personal care difficulties, even when she was still living on her own at that time.

Neurological examination showed hypomimia, mild rigidity in the four limbs, global bradykinesia, and unbalanced gait. There 
was a mild and mixed (rest and action) tremor affecting the four limbs. Her Mini-Mental Status Examination reflected deficits in memory, while her performance on the Frontal Assessment Battery was notable for impairment in inhibiting automatic responses, verbal fluency, and Trail making tests A \& B. She also demonstrated deficits in the memory and verbal fluency sections of the Seven Minute Screen.

Laboratory tests were all normal. Baseline MRI showed mild diffuse atrophy of the cerebrum together with some small vessel lesions affecting periventricular and semioval white matter. Follow-up MRI (5 years later) showed more intense atrophy in the left frontal and temporal lobes (Figure 1A). Tc-99mHMPAO SPECT showed bilateral but asymmetric hypoperfusion (more on the left side) on frontotemporal lobes (Figure 1B). 18F-FDG-PET showed mild to severe hypometabolism on the left frontotemporal lobe junction, and mild hypometabolism on the left fronto-basal left anterior cingulum regions (Figure 1C). DaTSPECT was informed as for the normal density of presynaptic dopaminergic uptakers (Figure 1D). Some irregularities in the morphology of basal ganglia and increase of cortical uptake were present.

The final diagnosis was frontotemporal lobe dementiabehavioral variant-with parkinsonism. Even when the density of presynaptic dopaminergic uptakers in the DaTSPECT was normal, she was put on Levodopa without significative clinical changes.

\section{PERSPECTIVE}

\section{Clinical Observations}

Parkinsonism is a well-known manifestation of FTLD (28). To date, presentations of FTLD with motor or movement disorders include (1) frontal lobe degeneration (FTD) with motor neuron disease (FTD-MND), (2) corticobasal degeneration (CBD), and (3) progressive supranuclear palsy (PSP). CBS and PSP usually show parkinsonism. However, parkinsonism has also been reported as a relative finding in other subtypes of FTD apart from CBS and PSP. Indeed, parkinsonism is found in approximately $20-30 \%$ of patients with FTLD. Furthermore, parkinsonism can be seen in all FTLD subtypes, and it can even be an outstanding feature in some cases. Therefore, there is a need to investigate parkinsonism in FTLD to obtain a better understanding of the disease.

Regarding the clinical characteristics, features of parkinsonism in FTLD are variable. The classical akinetic-rigid syndrome
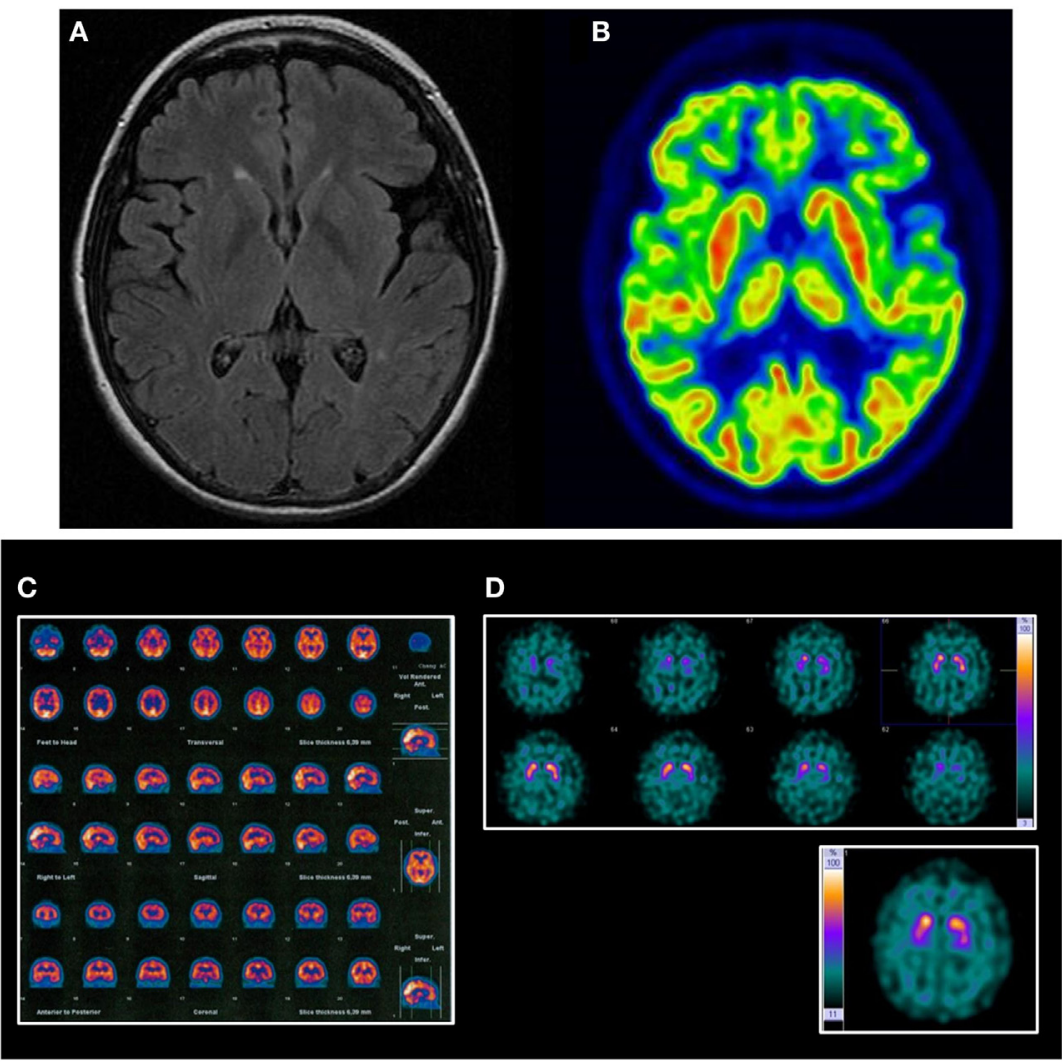

FIGURE 1 | Neuroimaging findings in the reported case. (A) Follow-up MRI mild diffuse atrophy with more intense atrophy on the left frontal and temporal lobes and some small vessel lesions affecting periventricular and semioval white matter. (B) FDG-PET shows mild-to-severe hypometabolism on the left frontotemporal lobe junction and mild hypometabolism on the left fronto-basal, left anterior cingulum regions. (C) HMPAO SPECT shows bilateral but asymmetric hypoperfusion (more on the left side) on frontotemporal lobes. (D) Dopamine transporter-single, photon emission computed tomography was informed of the normal density ofpresynaptic dopaminergic uptakers. Some irregularities in the morphology of basal ganglia and increase of cortical uptake can be noted. 
usually characterizes it. Other cases show atypical parkinsonism resembling PSP or CBD. Although rare, parkinsonism in FTLD may coexist with MND. Parkinsonism in FTLD is usually levodopa unresponsive, but there have been cases where a temporary benefit has been reported. The lack of response to levodopa reinforces the idea of a pathogenic mechanism different to PD.

\section{Genetics}

A systematic review aimed at the characterization of movement disorder phenomenology in genetically proven familial FTLD, showed that at any point during the disease, parkinsonism was the most common movement syndrome. It was reported in $79.8 \%$ of cases, followed by PSP and CBD syndromes in 12.2 and $10.7 \%$ of cases, respectively (28). The amyotrophic lateral sclerosis/parkinsonism dementia complex of Guam was probably the first known association of parkinsonism with dementia of frontotemporal features (29). Parkinsonism was frequently observed in familial FTLD, more specifically in FTLD with parkinsonism linked to chromosome 17q (FTDP17) (30). Parkinsonism in familial FTLD was first described in families with mutations in the microtubule-associated protein tau (MAPT) and progranulin (PRGN) genes. Since then, mutations in several other genes have been identified for FTLD with parkinsonism, including chromatin modifying protein 2B, chromosome 9 open reading frame 72 (C9ORF72), fused in sarcoma, valosin-containing protein, and transactive DNA-binding protein (TARDBP) $(31,32)$. Mutations in seven genes were robustly associated with autosomal dominant (SNCA, LRRK2, EIF4G1, VPS35) or recessive (parkin/PARK2, PINK1, DJ1/PARK7) PD or parkinsonism $(33,34)$. Changes in a long list of additional genes have also been suggested as causes for parkinsonism or PD, including genes for hereditary ataxias (ATXN2, ATXN3, FMR1), frontotemporal dementia (C9ORF72, GRN, MAPT, TARDBP), DYT5 (GCH1, TH, SPR), and others (ATP13A2, CSF1R, DNAJC6, FBXO, GIGYF2, HTRA2, PLA2G6, POLG, SPG11, UCHL1) (34-37).

\section{Neuropsychology and DaTSPECT Imaging in PD and FTLD}

Cognitive deficits could be identified in around a third of patients, even in the early untreated stages of PD (38-41). This cognitive dysfunction may have been related, in part, to reduced dopamine levels. While there were some inconsistent findings in the literature (42), fMRI studies with patients on and off levodopa indicated that higher levels of dopamine were associated with better cognitive performances on tasks of working memory and response accuracy (43). In addition, recent fMRI and DaTSPECT research have demonstrated a positive correlation between nigrostriatal dopaminergic function and performance on tests of executive functioning and memory (44). However, dopamine deficiency may not explain all of the cognitive deficits in PD, as some degree of cognitive impairment was common in patients diagnosed with related movement disorders such as dystonia (45) and essential tremor (46), conditions that were not associated with dopaminergic deficiency on imaging $(13,27,36)$. These patients usually had an abnormal DaTSPECT as the damage in frontostriatal neural circuitry occurred down (basal ganglia) to up (frontal cortex) (47).

It is a well-known fact that cognitive impairment in early PD and other synucleinopathies (Parkinson-plus syndromes) was accompanied by reductions in activity in frontostriatal neural circuitry $(48,49)$. There were five fronto-subcortical circuits linking different regions of the frontal cortex and subcortical nuclei, involving several neurotransmitters. Nigrostriatal dopaminergic neurons played an important role in some of these circuits, but gabaergic, glutamatergic, and cholinergic neurons were also extensively represented. Therefore, neurodegenerative disorders affecting neurons outside the nigrostriatal dopaminergic circuits were not be detected by DaTSPECTs and would have still been able to produce extrapyramidal symptoms.

When dementia preceded $\mathrm{PD}$, international consensus recommended a diagnosis of these patients with Dementia with Lewy bodies (DLB). Nevertheless, most patients with DLB and extrapyramidal signs had abnormal DaTSPECTs. In fact, autopsy studies provided class I evidence of 123I-FP-CIT dopaminergic neuroimaging accurately identifying patients with DLB (50), although this could be normal in those without extrapyramidal signs.

What is most relevant to the hypothesis presented in this paper is the fact that many SWEDD patients exhibited cognitive dysfunction. Studies with data from the Parkinson's Progression Marker Initiative (PPMI) database, showed that SWEDD patients performed significantly worse in semantic fluency and processing speed when compared with healthy controls (51). Montreal Cognitive Assessment test (MoCA) scores showed that about one-third of the PPMI participants were clinically diagnosed with SWEDD, experienced a statistically significant cognitive decline over the relatively brief period of 2 years, and a higher proportion of participants with SWEDD than DaTSPECT-confirmed PD had cognitive decline. The participants in the SWEDD group were more than twice as likely (relative risk $=2.07$ ) than those in the dopamine-deficient group to fall below the MoCA cognitive impairment cutoff. Thus, recently diagnosed patients with SWEDD may have been an even more significant risk for cognitive decline than patients with DaTSPECT-confirmed earlystage PD $(52,53)$. Neuropsychological performances between the SWEDD and the PD resulted similar when comparing patients in all stages (54). Altogether, these results suggested that SWEDD should not be considered a benign finding, as a high proportion of patients could be expected to suffer a cognitive decline in the close future.

Some studies have shown decreased uptake of DaT in bilateral putamina in FTLD with a negative correlation between the uptake ratio and parkinsonian motor status (55). Another study by Morgan and colleagues found that around a third of the FTLD cases may have presented abnormal scan and a significant reduction in uptake in the putamen and the caudate (56). Therefore, patients with FTLD may have had a positive or a negative DaTSPECT. This result probably depends on the neuropathology and clinical subtype of FTLD.

For instance, a study with patients with non-fluent/agrammatic variant of primary progressive aphasia (nfvPPA) and a logopenic variant of PPA (lvPPA) without clinical parkinsonism 
at baseline, showed reduced striatal tracer uptake in nfvPPA patients prior to clinical parkinsonism, especially for those nfvPPA without AD biomarkers, suggesting subclinical nigrostriatal degeneration (57). All lvPPA patients had normal DaTSPECT. Patients with nfvPPA presented abnormal DaTSPECT, especially in the left hemisphere compared with controls. DaTSPECT in nfvPPA patients with normal progranulin and negative CSF AD biomarkers was also significantly reduced compared with lvPPA patients with positive CSF AD biomarkers, suggesting nigrostriatal degeneration does not usually appear in the context of AD pathology resent, but it can appear in the context of FTD pathologies. During follow-up, seven nfvPPA/bio-patients developed parkinsonism, six of them with baseline reduced 123I-FP-CIT uptake (57).

\section{FINAL REMARKS}

The literature shows many clinical, genetic, and neuropsychological studies linking parkinsonism and FTLD. Also, a good number of articles provide evidence of the distinct characteristics between $\mathrm{PD}$ and SWEDD, and on the cognitive impairment present in many SWEDD cases. Finally, DaTSPECT studies in FTLD found both positive and negative results.

We report the case of a patient diagnosed with a behavioral variant of frontotemporal lobe dementia who also has parkinsonism that was not attributable to vascular lesions, antidopaminergic drugs, or any other etiology. DaTSPECT was informed as normal, and therefore it might be considered SWEDD.

On this basis, we reinforce the observation that parkinsonism without evidence of nigrostriatal dopaminergic deficit can be present in FTLD patients. Even when most of them will also

\section{REFERENCES}

1. De Celis Alonso B, Hidalgo-Tobon SS, Menendez-Gonzalez M, SalasPacheco J, Arias-Carrion O. Magnetic resonance techniques applied to the diagnosis and treatment of Parkinson's disease. Front Neurol (2015) 6:146. doi:10.3389/fneur.2015.00146

2. Sandoval-Rincon M, Saenz-Farret M, Miguel-Puga A, Micheli F, AriasCarrion O. Rational pharmacological approaches for cognitive dysfunction and depression in Parkinson's disease. Front Neurol (2015) 6:71. doi:10.3389/ fneur.2015.00071

3. Arias-Carrion O, Poppel E. Dopamine, learning, and reward-seeking behavior. Acta Neurobiol Exp (Wars) (2007) 67:481-8.

4. Marek K, Innis R, Van Dyck C, Fussell B, Early M, Eberly S, et al. [123I]betaCIT SPECT imaging assessment of the rate of Parkinson's disease progression. Neurology (2001) 57:2089-94. doi:10.1212/WNL.57.11.2089

5. Marek K, Jennings D, Seibyl J. Imaging the dopamine system to assess disease-modifying drugs: studies comparing dopamine agonists and levodopa. Neurology (2003) 61:S43-8. doi:10.1212/WNL.61.6_suppl_3.S43

6. Perlmutter JS, Eidelberg D. To scan or not to scan: DaT is the question. Neurology (2012) 78:688-9. doi:10.1212/WNL.0b013e3182494c72

7. Ba F, Martin WR. Dopamine transporter imaging as a diagnostic tool for parkinsonism and related disorders in clinical practice. Parkinsonism Relat Disord (2015) 21:87-94. doi:10.1016/j.parkreldis.2014.11.007

8. Surasi DS, Peller PJ, Szabo Z, Mercier G, Subramaniam RM. Dopamine transporter SPECT imaging in Parkinson disease and dementia. PET Clin (2013) 8:459-67. doi:10.1016/j.cpet.2013.08.006

9. Nicastro N, Burkhard PR, Garibotto V. Scan without evidence of dopaminergic deficit (SWEDD) in degenerative parkinsonism and dementia with Lewy present cognitive impairment, parkinsonism may be the main reason for consultation, and a normal DaTSCAN can be found during the diagnostic process. Therefore, FTLD may account for a percentage of patients with SWEDD. This origin might contribute to the observation that SWEDD patients exhibit a higher risk of cognitive impairment, worse mood, and better olfaction than PD patients $(19,51)$. Other causes of SWEDD have been described, particularly dystonic tremor. Thus, SWEDD should be understood as a heterogeneous group of patients in which some cases (probably the most tremoric ones) may have dystonic tremor, and some others may have FTLD (probably those with outstanding frontal cognitive dysfunction).

Careful clinical assessment remains the cornerstone of the study of patients with FTLD with parkinsonism. However, rationale support of genetic studies, neuropsychological evaluation, and neuroimaging, including structural and functional techniques, will provide a more detailed and comprehensive picture.

\section{ETHICS STATEMENT}

Written informed consent was obtained from the patient for the publication of this case report.

\section{AUTHOR CONTRIBUTIONS}

All authors contributed equally.

\section{FUNDING}

OA-C is supported by CONACyT FOSISS 2015-2 (262327).

bodies: a prospective study. J Neurol Sci (2018) 385:17-21. doi:10.1016/j. jns.2017.11.039

10. Schneider SA, Edwards MJ, Mir P, Cordivari C, Hooker J, Dickson J, et al. Patients with adult-onset dystonic tremor resembling parkinsonian tremor have scans without evidence of dopaminergic deficit (SWEDDs). Mov Disord (2007) 22:2210-5. doi:10.1002/mds.21685

11. Batla A, Erro R, Stamelou M, Schneider SA, Schwingenschuh P, Ganos C, et al. Patients with scans without evidence of dopaminergic deficit: a longterm follow-up study. Mov Disord (2014) 29:1820-5. doi:10.1002/mds.26018

12. Nicastro N, Garibotto V, Badoud S, Burkhard PR. Scan without evidence of dopaminergic deficit: a 10-year retrospective study. Parkinsonism Relat Disord (2016) 31:53-8. doi:10.1016/j.parkreldis.2016.07.002

13. Gerasimou G, Costa DC, Papanastasiou E, Bostanjiopoulou S, Arnaoutoglou M, Moralidis E, et al. SPECT study with I-123-Ioflupane (DaTSCAN) in patients with essential tremor. Is there any correlation with Parkinson's disease? Ann Nucl Med (2012) 26:337-44. doi:10.1007/s12149-012-0577-4

14. Erro R, Schneider SA, Stamelou M, Quinn NP, Bhatia KP. What do patients with scans without evidence of dopaminergic deficit (SWEDD) have? New evidence and continuing controversies. J Neurol Neurosurg Psychiatry (2016) 87:319-23. doi:10.1136/jnnp-2014-310256

15. Stoessl AJ. Scans without evidence of dopamine deficiency: the triumph of careful clinical assessment. Mov Disord (2010) 25:529-30. doi:10.1002/ mds. 23138

16. Fahn S; Parkinson Study Group. Does levodopa slow or hasten the rate of progression of Parkinson's disease? J Neurol (2005) 252(Suppl 4):IV37-42. doi:10.1007/s00415-005-4008-5

17. Silveira-Moriyama L, Schwingenschuh P, O'donnell A, Schneider SA, Mir P, Carrillo F, et al. Olfaction in patients with suspected parkinsonism and scans 
without evidence of dopaminergic deficit (SWEDDs). J Neurol Neurosurg Psychiatry (2009) 80:744-8. doi:10.1136/jnnp.2009.172825

18. Nalls MA, Mclean CY, Rick J, Eberly S, Hutten SJ, Gwinn K, et al. Diagnosis of Parkinson's disease on the basis of clinical and genetic classification: a population-based modelling study. Lancet Neurol (2015) 14:1002-9. doi:10.1016/ S1474-4422(15)00178-7

19. Taylor S, Gafton J, Shah B, Pagano G, Chaudhuri KR, Brooks DJ, et al. Progression of nonmotor symptoms in subgroups of patients with nondopamine-deficient parkinsonism. Mov Disord (2016) 31:344-51. doi:10.1002/ mds. 26456

20. Marshall VL, Patterson J, Hadley DM, Grosset KA, Grosset DG. Two-year follow-up in 150 consecutive cases with normal dopamine transporter imaging. Nucl Med Commun (2006) 27:933-7. doi:10.1097/01.mnm.0000243374. $11260.5 \mathrm{~b}$

21. Seibyl J, Jennings D, Tabamo R, Marek K. Unique roles of SPET brain imaging in clinical and research studies. Lessons from Parkinson's disease research. Q J Nucl Med Mol Imaging (2005) 49:215-21.

22. Marek K, Seibyl J, Eberly S, Oakes D, Shoulson I, Lang AE, et al. Longitudinal follow-up of SWEDD subjects in the PRECEPT Study. Neurology (2014) 82:1791-7. doi:10.1212/WNL.0000000000000424

23. Davies RR, Kipps CM, Mitchell J, Kril JJ, Halliday GM, Hodges JR. Progression in frontotemporal dementia: identifying a benign behavioral variant by magnetic resonance imaging. Arch Neurol (2006) 63:1627-31. doi:10.1001/ archneur.63.11.1627

24. Gomez-Tortosa E, Serrano S, De Toledo M, Perez-Perez J, Sainz MJ. Familial benign frontotemporal deterioration with C9ORF72 hexanucleotide expansion. Alzheimers Dement (2014) 10:S284-9. doi:10.1016/j.jalz.2013.09.013

25. Dols A, Krudop W, Moller C, Shulman K, Sajatovic M, Pijnenburg YA. Late life bipolar disorder evolving into frontotemporal dementia mimic. Neuropsychiatr Dis Treat (2016) 12:2207-12. doi:10.2147/NDT.S99229

26. Llamas-Velasco S, Garcia-Redondo A, Herrero-San Martin A, Puertas Martin V, Gonzalez-Sanchez M, Perez-Martinez DA, et al. Slowly progressive behavioral frontotemporal dementia with C9orf72 mutation. Case report and review of the literature. Neurocase (2018) 24:68-71. doi:10.1080/13554794.2 018.1428353

27. Menendez-Gonzalez M, Tavares F, Zeidan N, Salas-Pacheco JM, AriasCarrion O. Diagnoses behind patients with hard-to-classify tremor and normal DaT-SPECT: a clinical follow up study. Front Aging Neurosci (2014) 6:56. doi:10.3389/fnagi.2014.00056

28. Gasca-Salas C, Masellis M, Khoo E, Shah BB, Fisman D, Lang AE, et al. Characterization of movement disorder phenomenology in genetically proven, familial frontotemporal lobar degeneration: a systematic review and meta-analysis. PLoS One (2016) 11:e0153852. doi:10.1371/journal. pone. 0153852

29. Lee SE. Guam dementia syndrome revisited in 2011. Curr Opin Neurol (2011) 24(6):517-24. doi:10.1097/WCO.0b013e32834cd50a

30. Wszolek ZK, Tsuboi Y, Ghetti B, Pickering-Brown S, Baba Y, Cheshire WP. Frontotemporal dementia and parkinsonism linked to chromosome 17 (FTDP-17). Orphanet J Rare Dis (2006) 1:30. doi:10.1186/1750-1172-1-30

31. Kim EJ, Kwon JC, Park KH, Park KW, Lee JH, Choi SH, et al. Clinical and genetic analysis of MAPT, GRN, and C9orf72 genes in Korean patients with frontotemporal dementia. Neurobiol Aging (2014) 35:1213.e13-7. doi:10.1016/j. neurobiolaging.2013.11.033

32. Che XQ, Zhao QH, Huang Y, Li X, Ren RJ, Chen SD, et al. Genetic features of MAPT, GRN, C9orf72 and CHCHD10 gene mutations in Chinese patients with frontotemporal dementia. Curr Alzheimer Res (2017) 14:1102-8. doi:10. 2174/1567205014666170426105713

33. Nuytemans K, Theuns J, Cruts M, Van Broeckhoven C. Genetic etiology of Parkinson disease associated with mutations in the SNCA, PARK2, PINK1, PARK7, and LRRK2 genes: a mutation update. Hum Mutat (2010) 31:763-80. doi:10.1002/humu.21277

34. Puschmann A. Monogenic Parkinson's disease and parkinsonism: clinical phenotypes and frequencies of known mutations. Parkinsonism Relat Disord (2013) 19:407-15. doi:10.1016/j.parkreldis.2013.01.020

35. Tanikawa S, Mori F, Tanji K, Kakita A, Takahashi H, Wakabayashi K. Endosomal sorting related protein CHMP2B is localized in Lewy bodies and glial cytoplasmic inclusions in alpha-synucleinopathy. Neurosci Lett (2012) 527:16-21. doi:10.1016/j.neulet.2012.08.035
36. Scholz SW, Bras J. Genetics underlying atypical parkinsonism and related neurodegenerative disorders. Int J Mol Sci (2015) 16:24629-55. doi:10.3390/ ijms161024629

37. Wong YC, Krainc D. alpha-synuclein toxicity in neurodegeneration: mechanism and therapeutic strategies. Nat Med (2017) 23:1-13. doi:10.1038/ nm.4269

38. Muslimovic D, Post B, Speelman JD, De Haan RJ, Schmand B. Cognitive decline in Parkinson's disease: a prospective longitudinal study. J Int Neuropsychol Soc (2009) 15:426-37. doi:10.1017/S1355617709090614

39. Pfeiffer HC, Lokkegaard A, Zoetmulder M, Friberg L, Werdelin L. Cognitive impairment in early-stage non-demented Parkinson's disease patients. Acta Neurol Scand (2014) 129:307-18. doi:10.1111/ane.12189

40. Stefanova E, Ziropadja L, Stojkovic T, Stankovic I, Tomic A, Jecmenica-Lukic M, et al. Mild cognitive impairment in early Parkinson's disease using the movement disorder society task force criteria: cross-sectional study in Hoehn and Yahr stage 1. Dement Geriatr Cogn Disord (2015) 40:199-209. doi:10.1159/ 000433421

41. Weintraub D, Simuni T, Caspell-Garcia C, Coffey C, Lasch S, Siderowf A, et al. Cognitive performance and neuropsychiatric symptoms in early, untreated Parkinson's disease. Mov Disord (2015) 30:919-27. doi:10.1002/ mds. 26170

42. Poletti M, Bonuccelli U. Acute and chronic cognitive effects of levodopa and dopamine agonists on patients with Parkinson's disease: a review. Ther Adv Psychopharmacol (2013) 3:101-13. doi:10.1177/2045125312470130

43. Mattay VS, Tessitore A, Callicott JH, Bertolino A, Goldberg TE, Chase TN, et al. Dopaminergic modulation of cortical function in patients with Parkinson's disease. Ann Neurol (2002) 51:156-64. doi:10.1002/ana.10078

44. Lebedev AV, Westman E, Simmons A, Lebedeva A, Siepel FJ, Pereira JB, et al. Large-scale resting state network correlates of cognitive impairment in Parkinson's disease and related dopaminergic deficits. Front Syst Neurosci (2014) 8:45. doi:10.3389/fnsys.2014.00045

45. Scott RB, Gregory R, Wilson J, Banks S, Turner A, Parkin S, et al. Executive cognitive deficits in primary dystonia. Mov Disord (2003) 18:539-50. doi:10.1002/mds.10399

46. Lombardi WJ, Woolston DJ, Roberts JW, Gross RE. Cognitive deficits in patients with essential tremor. Neurology (2001) 57:785-90. doi:10.1212/ WNL.57.5.785

47. Zgaljardic DJ, Feigin A. Neuroimaging of Parkinson's disease and atypical parkinsonism. Curr Neurol Neurosci Rep (2004) 4:284-9. doi:10.1007/ s11910-004-0053-1

48. Zgaljardic DJ, Borod JC, Foldi NS, Mattis P. A review of the cognitive and behavioral sequelae of Parkinson's disease: relationship to frontostriatal circuitry. Cogn Behav Neurol (2003) 16:193-210. doi:10.1097/00146965200312000-00001

49. Zgaljardic DJ, Foldi NS, Borod JC. Cognitive and behavioral dysfunction in Parkinson's disease: neurochemical and clinicopathological contributions. J Neural Transm (Vienna) (2004) 111:1287-301. doi:10.1007/ s00702-004-0178-z

50. Thomas AJ, Attems J, Colloby SJ, O'brien JT, Mckeith I, Walker R, et al. Autopsy validation of 123I-FP-CIT dopaminergic neuroimaging for the diagnosis of DLB. Neurology (2017) 88:276-83. doi:10.1212/WNL. 0000000000003512

51. Wyman-ChickKA, Martin PK, Minár M, Menéndez-GonzálezM,Erickson LO, Álvarez-Avellón T, etal. Neuropsychological Test Performance in Parkinsonism Without Dopaminergic Deficiency on [123I]-FP-CIT SPECT Imaging. J Int Neuropsychol Soc (2018) 24:1-6. doi:10.1017/S1355617718000164

52. Wyman-Chick KA. Verbal fluency in Parkinson's patients with and without bilateral deep brain stimulation of the subthalamic nucleus: a meta-analysis. J Int Neuropsychol Soc (2016) 22:478-85. doi:10.1017/ S1355617716000035

53. Wyman-Chick KA, Martin PK, Minar M, Schroeder RW. Cognition in patients with a clinical diagnosis of Parkinson disease and scans without evidence of dopaminergic deficit (SWEDD): 2-year follow-up. Cogn Behav Neurol (2016) 29:190-6. doi:10.1097/WNN.0000000000000107

54. Wyman-Chick KA, Martin PK, Weintraub D, Sperling SA, Erickson LO, Manning CA, et al. Selection of normative group affects rates of mild cognitive impairment in Parkinson's disease. Mov Disord (2018). doi:10.1002/ mds. 27335 
55. Rinne JO, Laine M, Kaasinen V, Norvasuo-Heila MK, Nagren K, Helenius H. Striatal dopamine transporter and extrapyramidal symptoms in frontotemporal dementia. Neurology (2002) 58:1489-93. doi:10.1212/WNL. 58.10 .1489

56. Morgan S, Kemp P, Booij J, Costa DC, Padayachee S, Lee L, et al. Differentiation of frontotemporal dementia from dementia with Lewy bodies using FP-CIT SPECT. J Neurol Neurosurg Psychiatry (2012) 83:1063-70. doi:10.1136/ jnnp-2012-302577

57. Gil-Navarro S, Lomena F, Cot A, Llado A, Montagut N, Castellvi M, et al. Decreased striatal dopamine transporter uptake in the non-fluent/agrammatic variant of primary progressive aphasia. Eur J Neurol (2013) 20:1459-e126. doi:10.1111/ene.12196
Conflict of Interest Statement: The authors declare that the research was conducted in the absence of any commercial or financial relationships that could be construed as a potential conflict of interest.

Copyright (C) 2018 Menéndez-González, Álvarez-Avellón, Salas-Pacheco, de Celis-Alonso, Wyman-Chick and Arias-Carrión. This is an open-access article distributed under the terms of the Creative Commons Attribution License (CC BY). The use, distribution or reproduction in other forums is permitted, provided the original author(s) and the copyright owner are credited and that the original publication in this journal is cited, in accordance with accepted academic practice. No use, distribution or reproduction is permitted which does not comply with these terms. 